\title{
Open innovation in medical ultrasonics
}

\author{
Shin-ichiro Umemura
}

(C) The Japan Society of Ultrasonics in Medicine 2013

Totally closed innovation is impossible in any area of engineering. In medical engineering, closed innovation is particularly difficult as compared with other areas of engineering, because advanced medicine and advanced technology cannot both be possessed at the same time by a single person nor even a single group. From the mid-1970s to the early 1990s, Japanese industry was leading innovation in diagnostic ultrasound. A number of medically useful ultrasonic technologies were born in Japan. From engineers, innovation typically started as follows. An engineer or a group of engineers tackled a problem via private communication with medical doctors and/or in medical engineering conferences, and began developing technologies to solve the problem. From medical doctors, it may typically have started as follows. A medical doctor or a group encountered a problem in clinical practice, found an engineering approach, which seemed to solve the problem, from information in the public domain, and suggested developing related technologies, which really solve the problem, to an engineer or a group, mostly through private communication. Generations of prototypes were constructed by the engineers at the later stages of development, and typically these were tested by the medical doctors who had been involved at the earlier stages. The repeated feedback obtained through such tests was essential for the development for several reasons. The main reason is that the analog signals in a medical device were so difficult to quantify at each step at the necessary accuracy that purely quantitative design of the whole device was far from possible. Therefore, the innovation had to be partially closed between the engineers and medical doctors for a significant proportion of the development time. However, the structure of medical ultrasonic devices has changed since then. Ultrasonic signals have been mostly digitized at the front end since the mid-1990s. Most of the components, apart from ultrasonic probes, were basically standardized in the early 2010s. The development time for hardware is much shorter than it used to be. Therefore, the style of innovation must change accordingly. The group of people who establish the new optimal style will lead the innovation in this century. I would suggest to our colleagues significantly more open innovation in medical ultrasonics than in the past.

S. Umemura $(\bowtie)$

Graduate School of Biomedical Engineering, Tohoku University,

Miyagi, Japan

e-mail: sumemura@ecei.tohoku.ac.jp 